\title{
Effect of the Aedes fluviatilis Saliva on the Development of Plasmodium gallinaceum Infection in Gallus (gallus) domesticus
}

\author{
Ana CVM da Rocha/*, Érika M Braga*, Márcio SS Araújo, Bernardo S Franklin, \\ Paulo FP Pimenta/ ${ }^{+}$
}

\begin{abstract}
Laboratório de Entomologia Médica, Centro de Pesquisas René Rachou-Fiocruz, Av. Augusto de Lima 1715, 30190-002 Belo Horizonte, MG, Brasil *Departamento de Parasitologia, Universidade Federal de Minas Gerais, Belo Horizonte, MG, Brasil
\end{abstract}

Effect of Aedes fluviatilis saliva on the development of Plasmodium gallinaceum experimental infection in Gallus (gallus) domesticus was studied in distinct aspects. Chickens subcutaneously infected with sporozoites in the presence of the mosquito salivary gland homogenates $(S G H)$ showed higher levels of parasitaemia when compared to those ones that received only the sporozoites. However, the parasitaemia levels were lower among chickens previously immunized by SGH or non-infected mosquito bites compared to the controls, which did not receive saliva. High levels of anti-saliva antibodies were observed in those immunized chickens. Moreover, 53 and $102 \mathrm{kDa}$ saliva proteins were recognized by sera from immunized chickens. After the sporozoite challenge, the chickens also showed significant levels of anti-sporozoite antibodies. However, the ability to generate anti-sporozoites antibodies was not correlated to the saliva immunization. Our results suggest that mosquito saliva components enhance $\mathrm{P}$. gallinaceum parasite development in naive chickens. However, the prior exposure of chickens to salivary components controls the parasitemia levels in infected individuals.

Key words: saliva - avian malaria - mosquitoes - sporozoites - antibodies

Saliva components from haematophagous arthropods may modulate the host immune system and would represent an adaptation to evolution related with the blood feeding behavior (Ribeiro et al. 1984, Ribeiro 1987, 1989, James \& Rossignol 1991). Several substances in saliva from different vectors such as ticks, phlebotomine, and mosquitoes prevent host homeostasis at the bite site during the blood ingestion. Such components may show different activities such as anti-homeostatic, vasodilator, anti-inflammatory, immune-suppressor, and many others (review in Kamhawi et al. 2000).

The effect of saliva substances on the pathogen infectivity to vertebrates was firstly demonstrated by Titus and Ribeiro (1988) in phlebotomine followed by Junes and collaborators (1993) in ticks. Nowadays, the importance of saliva for blood feeding and pathogen infections has been extensively analyzed in several arthropod vectors (review in Gillespie et al. 2000). Therefore, there are few studies about the effect of mosquito saliva on infectivity of Plasmodium parasites. Early studies showed some protection against infection by $P$. berghei sporozoites when mice were previously immunized with mosquito salivary gland homogenate (Alger et al. 1972, Alger \& Harant 1976). In addition, Vaughan et al. (1999) suggested that saliva was one of the factors that could contribute to a more efficient rodent infection when the parasites were injected by mosquito vector bites.

Financial support: Fiocruz-Papes, CNPq, Pronex and Fapemig ${ }^{+}$Corresponding author: Fax: +55-31-3295.3115. E-mail: pimenta@cpqrr.fiocruz.br

Received 19 April 2004

Accepted 15 September 2004
In the current study, we evaluate the role of Aedes fluviatilis saliva in the experimental infections of chicken Gallus (gallus) domesticus with P. gallinaceum, the causative agent of avian malaria and its immunogenic potential to control parasitemia.

\section{MATERIALSAND METHODS}

Mosquitoes - Ae. fluviatilis were obtained from a colony established and kept in the Laboratory of Medical Entomology, Centro de Pesquisas René Rachou-Fiocruz in the state of Minas Gerais. The mosquitoes were kept in an acclimated insectary with average temperature between $26-28^{\circ} \mathrm{C}$ and relative air humidity around $70-80 \%$, in a cycle of $12 \mathrm{~h}$ in the dark and $12 \mathrm{~h}$ in the light (Consoli \& Williams 1978). The mosquitoes were provided with $10 \%$ glucose solution and water until the time of the experiments.

Mosquito infection - Groups of 4 to 6 day-old female mosquitoes were allowed to feed on the skin of $P$. gallinaceum infected chickens (parasitaemia levels ranging from 3.5 to $10 \%$ ). The infection evaluation was carried out 7 to 9 days after the infective blood meal by the presence of ookinetes in dissected midguts, which were dissected in PBS (phosphate buffer solution) and stained with $2 \%$ mercury chrome in order to visualize the parasites. The percentage of infected mosquitoes and the number of ookinetes per midgut were recorded through optical microscopic examination. All mosquitoes used to infect chickens were also previously examined for the presence of sporozoites 14 days after the infection (Ozaki et al.1984).

Chickens - Newborn G. (gallus) domesticus were acquired from a small commercial farm and maintained in cages. In order to avoid contact of the chickens with any arthropod before and during the experiments, the cages were wrapped with a transparent cotton fabric all the time. 
Salivary gland homogenate (SGH) - Salivary glands from 4 to 6 day-old female mosquitoes (non-infected mosquitoes) were dissected and transferred to microcentrifuge tubes containing $50 \mu \mathrm{l}$ cold sterile PBS. Dissected glands were stored at $-70^{\circ} \mathrm{C}$ until use when they were submitted to a successive freezing and unfreezing processes in order to obtain protein material. Total soluble protein concentration in the SGH was determined by Lowry method (Lowry et al. 1951). One salivary gland (a paired structure) dissected from one mosquito was estimated to contain $1.4 \mu \mathrm{g}$ of proteins.

Isolation of sporozoites - The P. gallinaceum sporozoites were obtained according to Ozaki et al. (1984). Briefly, 15 thoraces were dissected from 12-day infected mosquitoes and transferred to $0.5 \mathrm{ml}$ microcentrifuge-tube, containing fiberglass and with perforated bottom, and then placed into another $1.5 \mathrm{ml}$ microcentrifuge-tube. A volume of 50-100 $\mu 1$ of RPMI 1640 culture medium (Sigma, St. Louis, MD, US) supplemented with $10 \%$ chicken serum (for experimental infection) or PBS (for antigen preparation) was added to the thoraces. The microcentrifugetubes were centrifuged (twice, for $10 \mathrm{~min}$, at $1000 \mathrm{~g}$ ). The sediment was homogenized and parasites counted into Neubauer's chamber using a phase contrast microscope. The whole process was performed in ice to keep the sporozoite viability.

Infection of chickens with P. gallinaceum - One weekold chickens were used to evaluate the infection by $P$. gallinaceum sporozoites. Groups of five individuals were infected according to the following approaches: (i) naturally infected chickens infected by, bites of 10 infected mosquitoes; (ii) chickens infected by subcutaneous inoculation of $10^{3}$ sporozoites; (iii) infected chickens by subcutaneous inoculation of $10^{3}$ sporozoites added with SGH (corresponding to 10 mosquito glands); and (iv) noninfected chickens which were subcutaneous inoculated with RPMI 1640 culture medium. The subcutaneous via was chosen due to the early development of avian malaria into the phagocyte cells in the host skin at the infective bite site (Paraense 1941, 1943, Huff \& Coulston 1944, Coulston \& Huff 1947). Groups (i) and (iv) were considered as positive and negative controls of the infection.

Pre-patent periods (PPP), parasitaemia averages through blood smears stained with Giemsa solution and mortality rates were analyzed and the values compared throughout infection period in the studied groups of chickens. PPP is the period of time between the beginning of the infection and the time that the pathogen is detectable in the peripheral blood.

Saliva immunization - The effect of previous exposure to saliva components on the development of infection was analyzed in chickens which received bites from non-infected mosquitoes or which were inoculated by SGH. Groups of 5 or 10 chickens were used according to the following experimental approaches: (i) chickens immunized by bites of 10 non-infected adult mosquitoes; (ii) chickens immunized by subcutaneous inoculations of SGH (corresponding to 10 mosquito glands); and (iii) chickens which received PBS by subcutaneous inoculation. All chickens were one-week old in the beginning of the experiments and they were incubated once a week during a period of 4 or 7 weeks. Sera samples from the chickens were weekly obtained and stored at $-20^{\circ} \mathrm{C}$ until use. One week after the last incubated step, all the chickens received were infected by bites of 10 infected mosquitoes.

Detection of anti-sporozoite and anti-SGH IgG - Indirect immunofluorescence assay (IFA) were used to detect anti-sporozoite antibodies and Enzyme linked absorbance (ELISA) were used to detect anti-sporozoite and anti-saliva antibodies in infected or immunized chickens. Briefly, sporozoites were suspended in a concentration of $1 \times 10^{6}$ parasites $/ \mathrm{ml}$ and $5 \mu 1$ were deposited per well on slide. For screening, each well was incubated with $10 \mu$ of sera dilutions (1:20 to 1:5120) in PBS, and then incubated for $20 \mathrm{~min}$ with rabbit anti-chicken $\mathrm{IgG}$ fluorescein-conjugated (Funed) diluted 1:400 with PBS. The slides were examined by fluorescence microscopy. The ELISA were performed using high binding 96-well microplates (Nunc Maxisorp, Dynatech Denmark) covered with sporozoites homogenate or SGH $(20 \mu \mathrm{g} / \mathrm{ml})$ during $18 \mathrm{~h} 4^{\circ} \mathrm{C}$. Chicken sera were tested in triplicate at 1:80 dilution in PBS-Tween $(0.05 \%)$ for $2 \mathrm{~h}$ at $37^{\circ} \mathrm{C}$. The rabbit anti- chicken IgG peroxidase conjugated (Funed) was added at a 1:1000 dilution for $60 \mathrm{~min}$ at $37^{\circ} \mathrm{C}$ followed by addition of peroxidase substrate (OPD-O-phenylenediamine and hydrogen peroxide). Absorbance values were measured at $490 \mathrm{~nm}$. SGH and sporozoite homogenates were also fractionated by electrophoresis SDS-PAGE 12.5\% (Laemmli 1970) and then transferred to a nitrocellulose membrane (Amershan Pharmacia Hybond ${ }^{\mathrm{TM}}$ - C pure). The transference was performed using a $25 \mathrm{~V}$ constant voltage for $2 \mathrm{~h}$. Part of the nitrocellulose membrane containing the molecular markers was stained with Ponceau $\mathrm{S}$ for the visualization of the transferred proteins. The membrane with SGH or sporozoite proteins was incubated for $12 \mathrm{~h}$ with chicken sera diluted at 1:40. Afterwards, rabbit anti-chicken IgG peroxidase-conjugated (Funed) was added at a dilution of $1: 1000$. The antibody reaction was revealed by adding $0.05 \%$ 3.3-diaminobenzidin solution containing $0.025 \%$, 4-clhoride 1-naphtol and $0.03 \% \mathrm{H}_{2} \mathrm{O}_{2}(30 \% \mathrm{v} / \mathrm{v})$. The reaction was interrupted with distillated water after visualizing bands.

Statistical analysis - Average parasitemia ands average absorbance were compared using Student's $t$-test assuming equal variances. Differences in mortality between groups were tested with Chi-square test with Yates' correction. Difference was considered significant when Pvalue was less than 0.05 .

\section{RESULTS}

Effect of the SGH on avian infection - Two distinct experiments were conducted to analyze the effect of the saliva components in the infection of chickens with $P$. gallinaceum sporozoites (Fig. 1).

The parasitemia average among chickens that were infected by sporozoite inoculation in the presence of SGH was higher than those that did not receive SGH (Fig. 1). However, such tendency was not statistically different due to the significant deaths caused by malaria among chickens that were inoculated with parasites plus SGH.

The mortality rate of the chickens naturally infected 
by the mosquito bites was $100 \%$ up to the 13 day after infection. Therefore, the mortality rate of chickens infected with sporozoites in the presence of SGH was observed after the 12 day after infection and those infected ones without $\mathrm{SGH}$, only after the 15 day. It is important to point out that approximately $60 \%$ of chickens of the group infected by sporozoites inoculation (without SGH) and 40\% of chickens of the group infected by sporozoites with $\mathrm{SGH}$, controlled their parasitemia levels at the 18 day after infection (data not shown).

The IPPP among chickens naturally infected by mosquito bites was 5 days. Therefore, the PPPs for chickens infected by subcutaneous sporozoite inoculations with or without SGH were around 10 days (data not shown) showing no difference between the two groups.

Chicken infection after saliva immunization - Chickens that were immunized 7-fold with saliva by bites or inoculation of SGH showed similar PPP average values after a natural infection by mosquito bites. The average of the parasitaemia levels analyzed at the 9 day after infection of those immunized chicken groups showed to be lower than non-immunized chickens. Statistically significant differences of parasitaemia averages were observed at the 11 day after infection among the groups of immunized chickens and the control group (non-immunized chickens) (Table). The chickens that were immunized four times, either by the mosquito bites or by inoculation of $\mathrm{SGH}$, showed to be more susceptible to the infection than
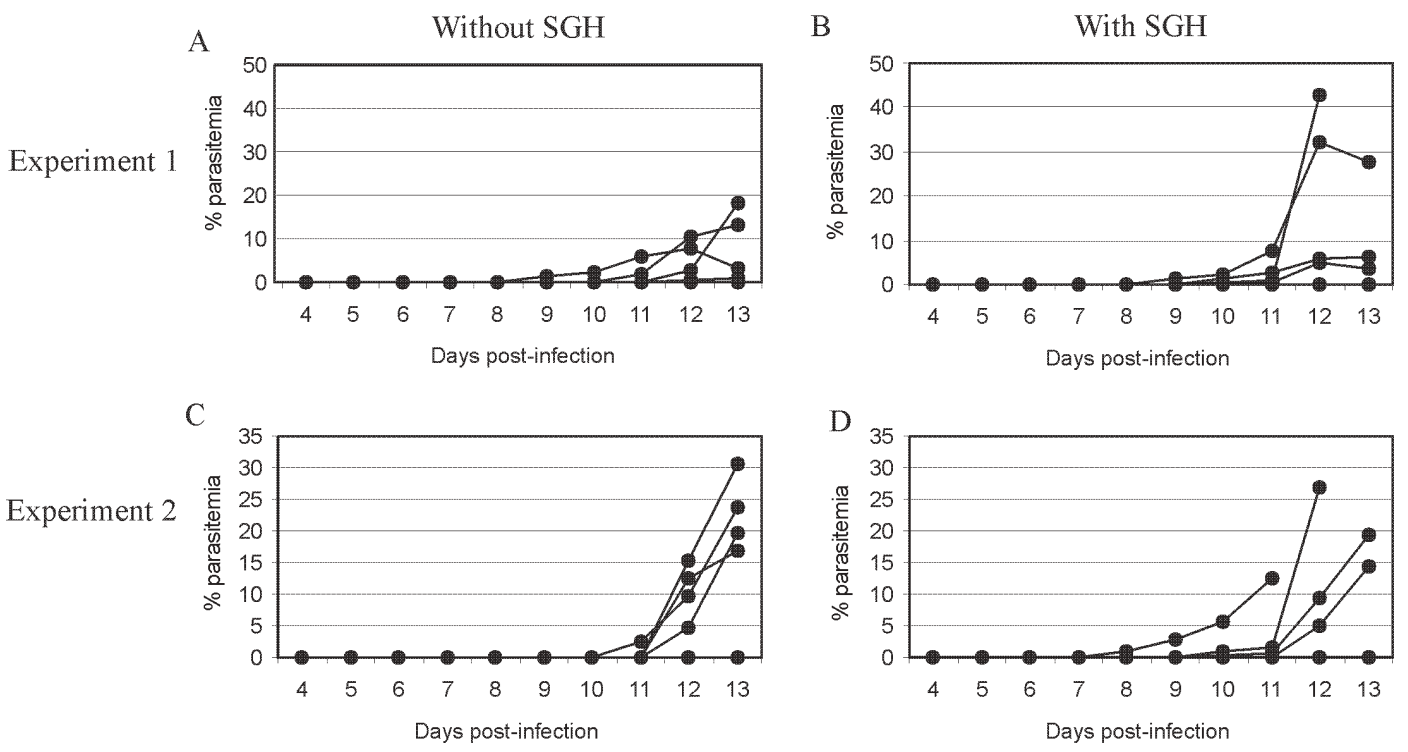

Fig. 1: the role of Aedes fluviatilis salivary gland homogenates (SGH) in individual parasitemias of Plasmodium gallinaceum infected chickens. Each group was inoculated with $10^{3}$ sporozoites in the presence (B and D) or in absence (A and C) of SGH.

TABLE

The role of immunization of the chicken with Aedes fluviatilis saliva in the parasitaemia and pre-patent period of Plasmodium gallinaceum infection

\begin{tabular}{|c|c|c|c|c|c|c|}
\hline \multirow[b]{2}{*}{ Groups ${ }^{a}$} & \multicolumn{5}{|c|}{ Parasitemia (X \pm d.p. $)^{b}$} & \multirow[b]{2}{*}{$\mathrm{PPP}^{c}$} \\
\hline & 7 day & 8 day & 9 day & 10 day & 11 day & \\
\hline $\begin{array}{l}\text { 4-fold immunizations by } \\
\text { mosquito bites }(n=5)\end{array}$ & $2.54 \pm 2.19$ & $4.02 \pm 2.11$ & $11.66 \pm 6.22$ & $4.66 \pm 5.04$ & $2.13 \pm 2.14$ & $5.00 \pm 0.55$ \\
\hline $\begin{array}{l}\text { 4-fold immunizations by } \\
\text { SGH }(n=5)\end{array}$ & $3.04 \pm 2.60$ & $5.00 \pm 3.08$ & $8.06 \pm 2.53$ & $3.58 \pm 2.25$ & $4.87 \pm 1.94$ & $6.00 \pm 0.55$ \\
\hline $\begin{array}{l}\text { 4-fold inoculations with } \\
\text { PBS (control) }(\mathrm{n}=5)\end{array}$ & $2.12 \pm 1.94$ & $5.00 \pm 5.27$ & $12.60 \pm 10.85$ & $10.80 \pm 12.66$ & $6.6 \pm 10.87$ & $6.00 \pm 0.89$ \\
\hline $\begin{array}{l}7 \text {-fold immunizations by } \\
\text { mosquito bites }(\mathrm{n}=10)\end{array}$ & $0.04 \pm 0.04$ & $0.80 \pm 0.74$ & $2.98 \pm 3.32$ & $4.28 \pm 2.76$ & $+2.94 \pm 3.72$ & $6.50 \pm 0.71$ \\
\hline $\begin{array}{l}\text { 7-fold immunizations by } \\
\text { SGH }(n=10)\end{array}$ & $0.04 \pm 0.007$ & $1.26 \pm 1.45$ & $6.80 \pm 7.15$ & $4.76 \pm 5.53$ & $+3.61 \pm 4.43$ & $7.00 \pm 0.83$ \\
\hline $\begin{array}{l}\text { 7-fold inoculations with } \\
\text { PBS (control) }(n=10)\end{array}$ & $0.02 \pm 0.04$ & $0.67 \pm 0.85$ & $5.59 \pm 5.94$ & $10.21 \pm 8.38$ & $11.43 \pm 8,66$ & $7.00 \pm 0.42$ \\
\hline
\end{tabular}

$\mathrm{P}<0,05 ; a$ : chickens from each group were challenged by infected mosquito bites; $b$ : percentage of infected red blood cells after the challenge; $c$ : interval between the time of the challenge and the parasite appearance in the peripheral blood; SHG: salivary gland homogenates 
those chickens 7-fold immunized (data not shown).

In a follow up of the infection rates during one month, the chickens naturally immunized by mosquito bites presented an average of the mortality rates of $10.5 \%$. Chickens from the SGH-immunized and non-immunized groups showed mortality rates of $12 \%$ and $13 \%$, respectively. All the chickens that remained alive in these studied groups appeared to have controlled parasitaemia revealed by negative microscopy examination of blood smears.

Anti-sporozoite antibodies in P. gallinaceum infected chickens - Anti-sporozoite antibodies in chicken sera before and after infections were also evaluated. Anti-sporozoite antibodies were not detected by IFA in sera from one-week chickens infected with $P$. gallinaceum. Fig. 2 shows the individual and the average of sera absorbance values by ELISA verified in a representative experiment. A raise in average values can be observed comparing sera from chickens before and 10 days after infection, independent on the infection via. However, absorbance values average among the experimental groups showed to be relatively low (lower than 0.4 ). Only two sera from chickens infected by sporozoites with SGH had individual absorbance values higher than 0.6 (Fig. 2).

Anti-sporozoite antibodies in saliva-immunized chickens infected with P. gallinaceum - Presence of antisporozoite antibodies was not detected among non-infected saliva-immunized chickens. On the other hand, after infection by mosquito bites, all groups of chicken showed anti-sporozoite antibodies including the non-immunized control group. Anti-sporozoite IgG antibodies titles detected by IFA, ranged from 1:40 to 1:1280 in chickens 4-fold immunized reaching a high value $(1: 2560)$ in 7 fold immunized ones. No statistical difference was observed for antibody titles detected by IFA. However, anti-

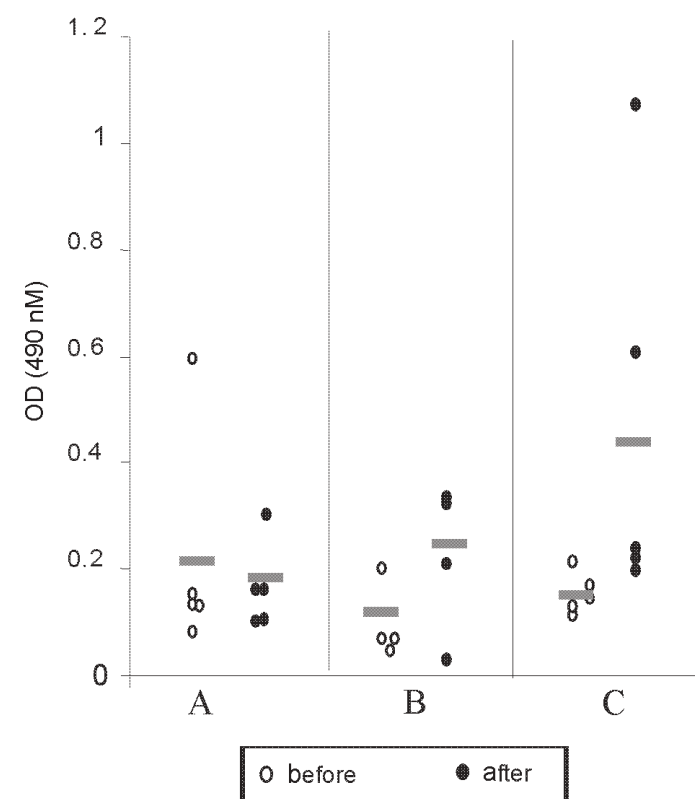

Fig. 2: ELISA reactivity for Plasmodium gallinaceum sporozoite antigens to sera from 1-week old chicken before $(O)$ and 10 days after infection (-) using different via of parasite inoculation (A: mosquito bites; B: inoculation of sporozoites; C: inoculation of sporozoites plus SGE). Bars: average of the absorbance values. sporozoite antibody frequencies and proportions detected by ELISA showed a statistically significant raise in the average of the absorbance values, which were mainly observed 10 days after infection (Fig. 3).

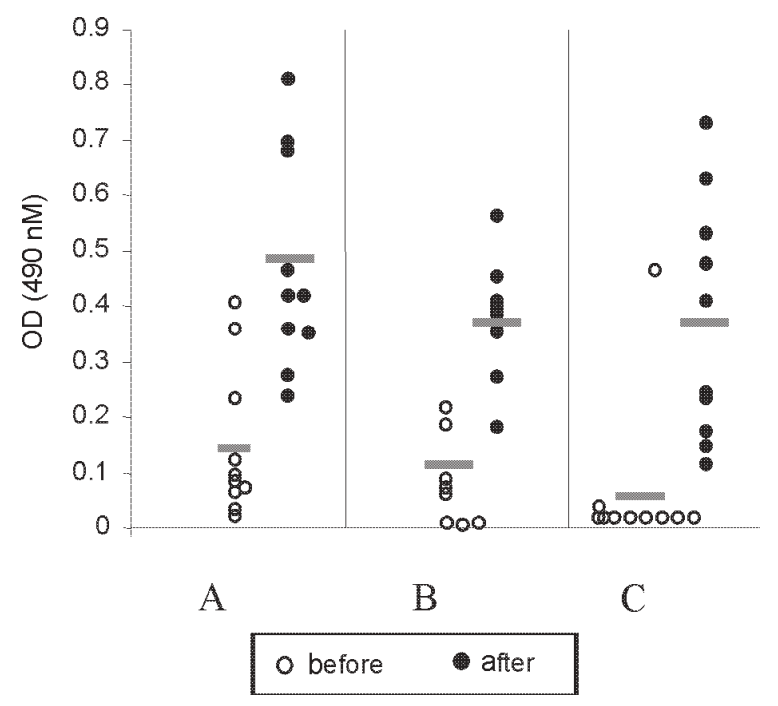

Fig. 3: ELISA reactivity for Plasmodium gallinaceum sporozoite antigens to sera from chickens which were saliva-immunized by mosquito bites (A) or salivary gland homogenates inoculation (B) or non-immunized control $(\mathrm{C})$. The experiments were done before (O) and 10 days after infection $(0)$ by mosquito bites. Bars: average of the absorbance values.

Anti-saliva antibodies in P. gallinaceum infected chickens - Individual values of anti-antibody saliva absorbancies from chickens infected by the mosquito bites or by sporozoite inoculation with or without SGH, showed to be low (lower than 0.4 ) with no statistically differences among groups (not shown). However, 10 days after infection was observed significant increase in anti-saliva antibody levels among immunized chickens independently of the inoculation via. Such antibody raise were also verified for the control group (Fig. 4). In spite of the number of previous immunizations no statistically significant difference among groups was observed.

Recognition of antigenic proteins of P. gallinaceum sporozoite and salivary gland by Western blotting - Sera from chickens infected by $P$. gallinaceum bites, either from the group of previous saliva-immunized chickens (SGH or mosquito bites) or control group (non-immunized chickens), recognized sporozoite proteins with molecular weights of approximately 64 and $76 \mathrm{kDa}$ (Fig. 5). Which correspond to the $P$. gallinaceum $\mathrm{CS}$ protein.

All those sera from chickens of the same groups recognized a protein with a molecular weight of approximately $102 \mathrm{kDa}$ that is presented in SGH (Fig. 6). In addition, proteins of 53 and $59 \mathrm{kDa}$ present in $\mathrm{SGH}$ were recognized by the sera from immunized or non-immunized adult chickens infected by the mosquito bites.

\section{DISCUSSION}

The avian malaria infection with $P$. gallinaceum has been considered a good model for developing studies 


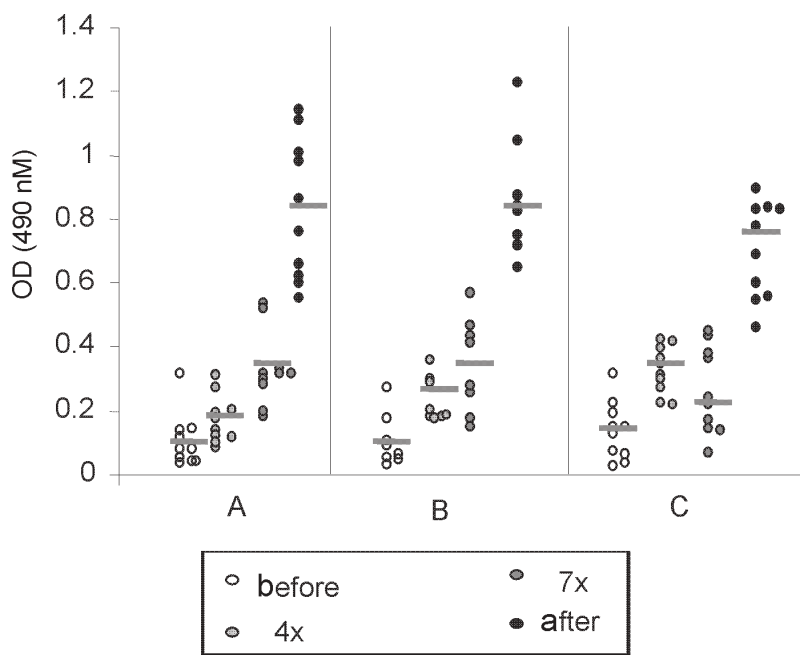

Fig. 4: ELISA reactivity against Aedes aegypti saliva antigens present in sera from chickens, which were saliva-immunized by mosquito bites (A) or salivary gland homogenates inoculation (B) or nonimmunized, control $(\mathrm{C})$. The experiments were done before the infection, 4-fold or 7-fold after immunizations and 10 days after infection by mosquito bites. The control group received only PBS.

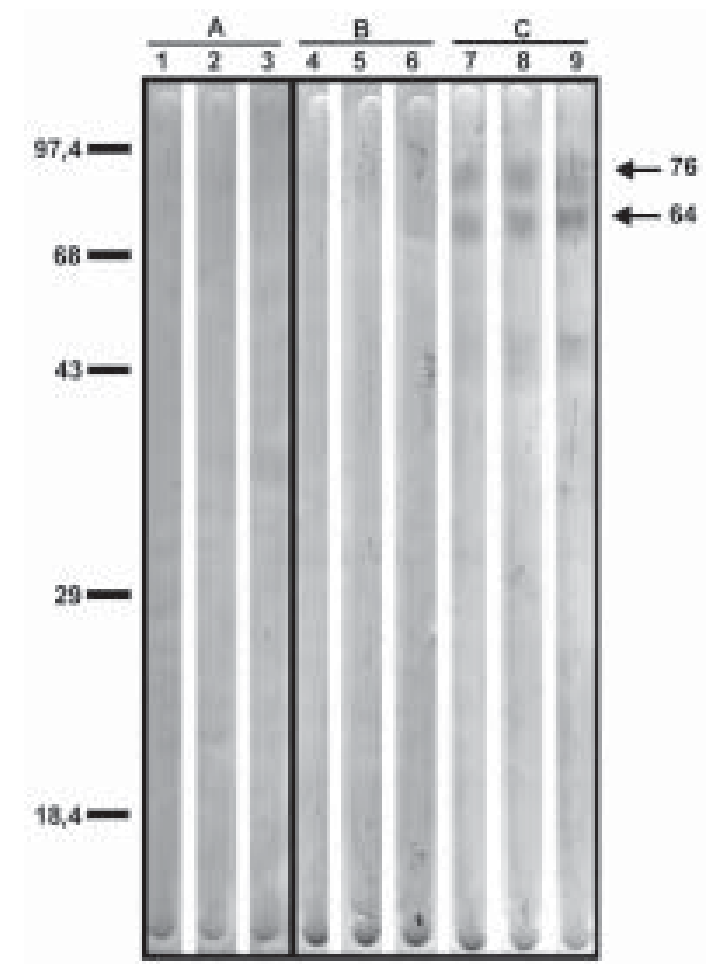

Fig. 5: Western-blotting showing reactivity against Plasmodium gallinaceum sporozoites antigens using sera from different groups of chickens. A: one-week old chickens infected with inoculation of $10^{3}$ sporozoites (1), $10^{3}$ sporozoites plus salivary gland homogenates (SGH) (2) and mosquito bites (3); B: 4-week old chickens immunized with saliva by SGH inoculations (4); mosquito bites; (5) control (inoculated with PBS) (6); C: 4-fold saliva-immunized chickens followed by infection with mosquito bites, which were immunized by salivary gland homogenates inoculations (7) or mosquito bites (8) and control (inoculated with PBS ) (9).

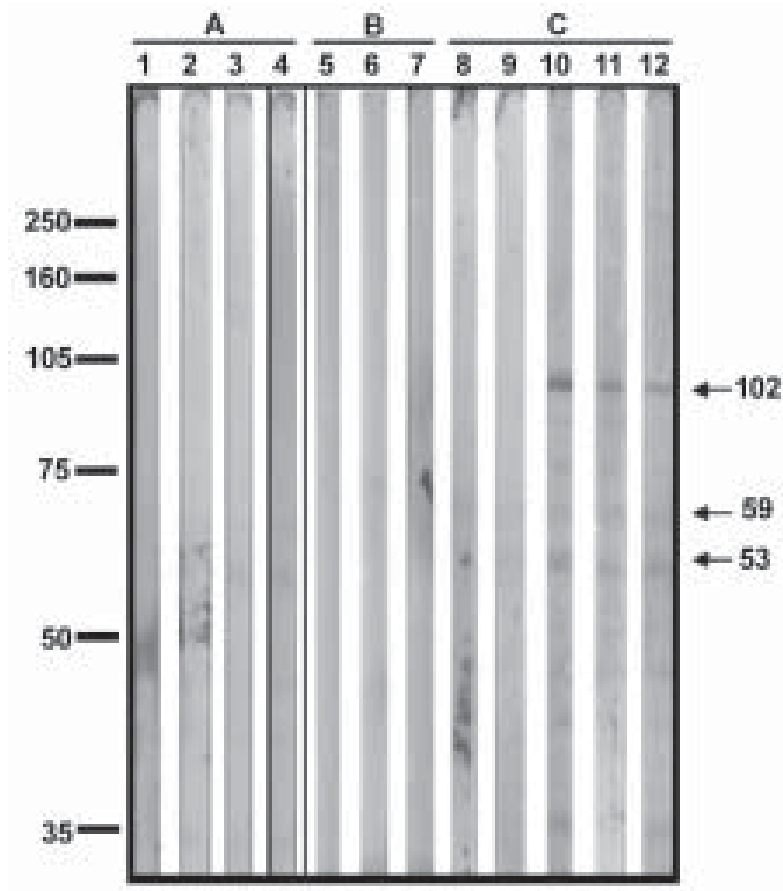

Fig. 6: Western-blotting showing reactivity against Aedes fluviatilis saliva antigens using sera from different groups of chickens. A: oneweek old chickens infected with inoculation of $10^{3}$ sporozoites (1), $10^{3}$ sporozoites plus salivary gland homogenates (SGH) (2), mosquito bites (3), and PBS (control non-infection), (4); B: 4-week old chickens immunized four times with SGH inoculations (5), mosquito bites (6), and control (inoculations with PBS) (7); C: 7-fold salivary gland homogenates immunized chickens; (8) or mosquito bites (9) which were respectively infected by mosquito bites (10 and 11) and control, non-immunized chickens but infected by mosquito bites (12).

related with the parasite-host interaction processes. In mental subcutaneous sporozoite inoculation as previously observed (Vaughan et al. 1999). In addition, it is important to consider that sporozoites isolated from salivary glands are considered an heterogeneous population. Moreover, sporozoites need to pass through several biological processes inside the salivary glands in order to reach the salivary duct (Pimenta et al. 1994). Although there are few thousands of sporozoites stocked up in the salivary gland, only a small number is ready to be injected by the mosquito bites (Simonetti 1996). Certainly, not all sporozoites obtained from the salivary gland by the isolation procedure are able to stay alive and develop infection in the skin host.

Considering that age-dependent resistance has been demonstrated for pathogens (Kogut et al. 1998, 2002), we evaluated the susceptibility to infection in chickens with different ages. Our results indicate some effect of host age on $P$. gallinaceum infection: adult chickens showed a prepatent period higher than that observed for young chickens. The young chickens developed the infection in a very quick way with a great number of parasites in the peripheral blood being fatal for several individuals. In opposite, adult chickens appear to be less susceptible to the avian malarial parasite controlling the infection with 
the absence of deaths. These facts were only observed in infections caused by mosquito bites and not by subcutaneous inoculation of sporozoites, once again, confirming the efficacy of the natural via of infection.

It is interesting to note that the presence salivary components in the sporozoite inoculum affect the parasitaemia levels and the mortality rates. An increase in parasitemia levels and in mortality rates was observed when chickens received sporozoites in association with SGH. However, prepatent period, was not affect by the saliva components present in the subcutaneous inoculum. Our results obtained for $P$. gallinaceum infection, corroborate literature data concerning other pathogens, which evidence the effects of arthropod saliva in parasite-vector interactions (Ribeiro et al. 1985, Titus \& Ribeiro 1988, Belkaid et al. 1998, Kamhawi et al. 2000).

In our experiments, anti-sporozoite antibodies were detected in chickens infected with $P$. gallinaceum by mosquito or subcutaneous inoculation. Lower levels of these antibodies were detected in young individuals comparing with the adults. The chickens showed to produce anti-sporozoite antibodies with the same molecular (64 $\mathrm{kDa}$ and $76 \mathrm{kDa}$ ) weights of the well-known circumsporozoite protein family, which covers the surface of the Plasmodium sporozoite (CS protein). The $P$. gallinaceum CS protein is involved in parasite interaction with vertebrate and invertebrate cell hosts and elicits a strong humoral response in chicks (Daher \& Krettli 1987). In the present study, we demonstrated that adult chickens infected by the mosquito bites produced anti-SGH antibodies. During the blood meal, female mosquitoes deposit saliva into the host skin. Adult chickens sera recognized some SGH proteins including one that correspond to the molecular weight of apirase $(64 \mathrm{kDa})$. Apirases are enzymes that have been demonstrated in the saliva of several insect vectors and are recognized as playing a role in the insect feeding process avoiding the blood platelet aggregation (Ribeiro 1987). Previous work already showed that anti-apirase antibodies in saliva-immunized mice by successive bites of the mosquito Anhopheles stephensi were able to inhibit apirase activity impairing the blood meal screening (Mathews et al. 1996).

The parasitaemia levels showed to be lower in immunized groups of chickens than in the control group. Alger and Harant (1976) also reported that immunized mice with mosquito salivary glands were protected against the $P$. berghei sporozoites challenge. Prior exposure of mice to bites of non-infected sand flies protects against Leishmania major (Kamhawi et al. 2000). However, the sand fly saliva components enhance the cutaneous lesion caused by the parasite. It appears that similar phenomena also occur in saliva-immunized chicken challenged with $P$. gallinaceum.

In conclusion, our results suggest that mosquito saliva components play an important role in the $P$. gallinaceum infection in chickens. Moreover, saliva also affects the course of $P$. gallinaceum infection in previously immunized chickens controlling the parasitemia levels. Thus, the role of saliva and its possible use for vaccination against pathogens could be considered. Saliva also can enhance transmission of parasites/pathogens by arthropods. As a result, vaccines that target the arthropod (e.g. salivary immunomodulators) should be considered as one component of multi-subunit vaccines against arthropod-borne pathogens.

\section{ACKNOWLEDGMENTS}

To Denise Nacif Pimenta and Paola Seabra Eiras for correcting the English version.

\section{REFERENCES}

Alger NE, Harant JA, Willi LC, Jorgensen GM 1972. Sporozoite and normal salivary gland induced immunity in malaria. Nature 238: 341-342.

Alger NE, Harant JA 1976. Plasmodium berghei: protection against sporozoite by normal mosquito tissue vaccination of mice. Exp Parasitol 40: 269-272.

Belkaid YS, Kamhawi S, Modi G, Valenzuela J, Trauth NN, Rowton E, Ribeiro J, Sacks DL 1998. Development of a natural model of cutaneous leishmaniasis: powerful effects of vector saliva and saliva pre-exposure on the long-term outcome of Leishmania major infection in the mouse ear dermis. J Exp Med 188: 1941-1953.

Coatney GR, Cooper WC, Miles VI 1944. Studies on Plasmodium gallinaceum Brumpt. I. The incidence and course of the infection in young chicks resulting from single mosquito bites. Am J Trop Med Hyg 41: 109-118.

Consoli RA, Willians GBP 1978. Laboratory observation on the bionomics of Aedes fluviatilis (Lutz, 1904) (Diptera: Culicidae). Bull Entomol Res 68: 123-136.

Coulston F, Huff CG 1947. The morphology of cryptozoites and metacryptozoite of Plasmodium relictum and the relationships of these stages to parasitemia in canaries and pigeons. J Infect Dis 80: 209-213

Daher VR, Krettli AU 1987. Experimental vaccination of chikens with Plasmodium gallinaceum sporozoites. I. Circumsporozoite proteins are expressed by sporozoites recovered from both salivary glands and midguts of mosquitoes. J Protozool 34: 245-249.

Gillespie RD, Mbow ML, Titus RG 2000. The immunomodulatory factors of blood feeding arthropod saliva. Parasit Immunol 22: 319-331.

Huff CG, Coulston F 1944. The development of Plasmodium gallinaceum from sporozoite to erytrocytic trophozoite. $J$ Infect Dis 75: 231-249.

James AA, Rossignol PA 1991. Mosquito salivary glands: parasitological and molecular aspects. Parasitol Today 7: 267271.

Junes LD, Kaufman WR, Nuttali PA 1993. Modification of the skin-feeding site by tick saliva mediates virus transmission. Experientia 48: 779-782.

Kamhawi S, Belkaid Y, Govind M, Rowton E, Sacks DL 2000. Protection against cutaneous leishmaniasis resulting from bites of uninfected sand flies. Science 290: 1351-1354.

Kogut MH, Lowry VK, Moyes RB, Bowden LL, Bowden R, Genovese K, Deloach JR 1998. Lymphokine augmented activation of avian heterophils poult. Science 77: 964-971.

Kogut M, Rothwell L, Kaiser P 2002. Differential effects of age on chicken heterophil functional activation by recombinant chicken interleukin-2. Dev Comp Immumol 26: 817-830.

Laemmli UK 1970. Cleavage of structural proteins during the assembly of the head of bacteriophage T4. Nature 227: 680-685.

Li X, Sina B, Rossignol PA 1992. Probing behavior and sporozoite delivery by Anopheles stephensi infected with Plasmodium berghei. Med Vet Entomol 6: 57-61. 
Lowry OH, Rosbrough NJ, Farr AL, Randall JR 1951. Protein measurement with the Folin phenol reagent. J Biol Chem 193: 265-275.

Mathews GV, Sidjanski S, Vanderberg JP 1996. Inhibition of mosquito salivary gland apyrase activity by antibodies produced in mice immunized by bites of Anopheles stephensi mosquitoes. Am J Trop Med Hyg 55: 417-423.

Mccutchan TF, Kissinger JC, Touray JC, Rogers MJ, Sullivan JL1M, Braga EM, Krettli AU, Miller LH 1996. Comparison of circumsporozoite proteins from avian and mammalian malarias. Biological and phylogenetic implications. PNAS (USA) 93: 11889-11894.

Mccutchan T F, Dame JB, Miller LH, Barnwell J 1984. Evolutionary relatedness of Plasmodium species as determined by structure of DNA. Science 225: 808-811.

Meis JFGM, Runtjes PJM, Verhave JP, Ponnudurai T, Hollingdale MR, Smith JE, Sinden RE, Kap PHK, Meuwissen JHETH, Yap SH 1986. Fine structure of malaria parasite Plasmodium falciparum in human hepatocytes in vitro. Cell Tiss Res 244: 245-350.

Ozaki LS, Gwads RW, Godson GN 1984. Simple centrifugation method for rapid separation of sporozoites from mosquitoes. J Parasitol 70: 831-833.

Pimenta PF, Touray M, Miller L 1994. The journey of malaria sporozoites in the mosquito salivary gland. Eukaryot Microbiol 41: 608-624.

Paraense WL 1941. Observações preliminares sobre o ciclo exoeritrocítário do Plasmodium juxtanucleare Versiani e Gomes. Mem Inst Oswaldo Cruz 45: 813-824.

Paraense WL 1943. Aspectos parasitários observados no local inoculado com esporozoítos de Plasmodium gallinaceum. Mem Inst Oswaldo Cruz, 38: 352-360.
Simonetti AB 1996. The biology of malarial parasite in the mosquito - A review. Mem Inst Oswaldo Cruz 91: 519-541.

Ribeiro JMC 1987. Role of saliva in blood-feeding by arthopods. Ann Rev Entomol 32: 463-478.

Ribeiro JMC 1989. Role of saliva in tick/host interactions. Exp Appl Acarol 7: 15-20.

Ribeiro JMC, Makoul G, Levine J, Robinson D, Spielman A 1985. Antihemostatic, antiinflamatory and immunosuppressive properties of the saliva of a tick, Ixodes dammini. J Exp Med 161: 332-344.

Ribeiro JMC, Rossignol P, Spielman A 1984. Role of mosquito saliva in blood vessel location. J Exp Biol 108: 1-9.

Rosenberg R, Rungsiwongse J 1990. The number of sporozoites produced by individual malaria oocysts. Am J Trop Med Hyg 45: 574-577.

Rosenberg R, Wirtz RA, Schneider I, Burge R 1991. An estimation of the number of malaria sporozoites ejected by feeding mosquito. Trans R Trop Med Hyg 84: 7725-7727.

Titus RG, Ribeiro JMC 1988. Salivary gland lysates from the sand fly Lutzomyia longipalpis enhance Leishmania infectivity. Science 239: 1306-130.

Vanderberg JP 1977. Plasmodium berghei: quantification of sporozoites injected by mosquitoes feeding on a rodent host. Exp Parasitol 42: 169-181.

Vaughan JA, Scheller LF, Wirtz RA, Azad AF 1999. Comparative infectivity of Plasmodium berghei sporozoites delivered by intravenous inoculation versus mosquito bite: implications for sporozoite vaccine trials. Infect Immun 67: 4285-4289.

Waters AP, Higgins DG, Mccutchan TF 1991. Plasmodium falciparum appears to have arisen as a result of lateral transfer between avian and human hosts. PNAS (USA) 88: 31403144. 
